УДК 621.78:669.14-022.532

\title{
ОСОБЕННОСТИ ЭВОЛЮЦИИ НАНОМАСШТАБНЫХ ХАРАКТЕРИСТИК МЕТАЛЛА ПРИ НАГРУЖЕНИИ ТЕРМИЧЕСКИМИ ЦИКЛАМИ
}

\author{
Заворин Александр Сергеевич1, \\ zavorin@tpu.ru
}

\author{
Любимова Людмила Леонидовна', \\ III@tpu.ru
}
Буваков Константин Владимирович 1 , buvakov@tpu.ru

Ташлыков Александр Анатольевич ${ }^{1}$, tashlykov@tpu.ru

\author{
Фисенко Роман Николаевич1, \\ ronifis@tpu.ru \\ 1 Национальный исследовательский Томский политехнический университет, \\ Россия, 634050, г. Томск, пр. Ленина, 30.
}

\begin{abstract}
Актуальность исследования обусловлена отсутствием экспериментальных данных, устанавливающих связь между субмикроскопическими свойствами (свойствами III рода) и макроскопическими свойствами I рода. Такая взаимосвязанность, являющаяся фрактором влияния на отношение «микроструктура-прочность», может способствовать дальнейшим успехам в решении вопросов ресурса.

Цель исследования заключается в установлении влияния циклических термических нагрузок на среднеквадратичные смещения атомов как показатель прочности межатомных связей и микроповрежденности металла пароперегревательных труб.

Объектом исследования являются образцы из околошовных зон однородного сварного шва, выполненного из жаропрочной перлитной стали $12 X 1 M \Phi$.

Методы: физическое моделирование условий эксплуатации путем воздействия на образцы циклами термической нагрузки в электропечи МИМП-10УЭ, рентгенометрия образцов, оценка внутренних структурных напряжений на рентгеновских дифррактометрах ДРОН, морфология поверхности с помощью портативного электронного микроскопа типа PENSCKOPE.

Результаты. Установлено влияние циклических термических нагрузок на среднеквадратичные смещения атомов. Показано, что в результате термического влияния прочность межатомных связей увеличивается. Проиллюстрирована корреляция между субмикро- и макроскопическими характеристиками прочности, и показано, что амплитуда тепловых колебаний атомов, являясь характеристикой прочности межатомных связей, может быть диагностическим признаком накопления и развития повреждаемости металла. Уменьшение амплитуды среднеквадратичных смещений атомов при термоциклировании объяснено на основе гипотезы о возникновении твердых растворов замещения в результате процесса азотирования поверхности молекулярным азотом атмоссрерного воздуха. В рамках этой гипотезы показано, что одним из механизмов осцилляции внутренних напряжений I рода при термоциклировании может быть процесс азотирования поверхности, приводящий к организации в многокомпонентных легированных сталях сложных связей «металл-неметалл», направленных на поддержание устойчивости системы твердых растворов.
\end{abstract}

Ключевые слова: Сварной шов, околошовная зона, среднеквадратичные отклонения атомов, внутренние напряжения, цикль термического нагружения.

\section{Введение}

Проблемы прочности, долговечности и коррозии элементов оборудования и сварных швов являются актуальными для современной теплоэнергетики [1-7 и др.]. Продление срока службы элементов и узлов теплоэнергетического оборудования все чаще становится разумной альтернативой техническому перевооружению [8-14 и др.]. С этой целью разрабатываются объемы и регламент ремонтных работ и технологии термовосстановления, направленные на регенерацию структуры материала [15-17 и др.].

Для описания условий и диагностики признаков возникновения предельного состояния металла, а также признаков, при которых возможно восстановление, используются представления о повреждаемо- сти. Повреждаемость проявляется различными изменениями структуры материала - сдвиговыми процессами внутри зерна, образованием двойников, дроблением зерен, образованием пористости, перекристаллизацией упрочняющих фаз и так далее, вызывая снижение ресурса. Поэтому для оценок поврежденности используют характеристики кратковременной и длительной прочности, ползучести, многоцикловой усталости, а также некоторые физические характеристики, которые в ряде случаев становятся мерой количественной оценки степени поврежденности материала (микротвердость, $\sigma_{\mathrm{B}}, \sigma_{0,2}$ ) [18-24 и др.].

Разрушение определяется процессами накопления повреждений на разных масштабных уровнях [25]:

- макроскопический уровень, связанный с разрушением тела (свойства I рода); 
- микроскопический уровень, обеспечиваемый прочностью микрочастицы (свойства II рода);

- субмикроскопические повреждения, связанные с прочностью межатомных связей, обеспечивающих сцепление в материале (свойства III рода).

Для контроля узлов теплоэнергетического оборудования в основном применяют методы, связанные со свойствами I и II рода, такие как радиографический, ультразвуковой, магнитопорошковый контроль, цветной и люминесцентный методы, капиллярная дефектоскопия и т. д.

Значительно слабее представлены экспериментальные данные, устанавливающие связь между субмикроскопическими свойствами и свойствами I рода [26-29 и др.].

Иллюстрацией субмикроскопических свойств могут быть экспериментальные зависимости среднеквадратичных смещений атомов при механических или термических нагрузках. При этом среднеквадратичные смещения и определяемая на их основе характеристическая температура Дебая, известная как показатель прочности межатомных связей, могут быть показателем микроповрежденности металла паропроводных труб и одновременно с этим служить признаком интенсивности процесса накопления предельных повреждений [30-35 и др.].

Наиболее повреждающими факторами при эксплуатации тепломеханического оборудования ТЭС являются нестационарные режимы работы оборудования [36, 37 и др.].

Поэтому цель настоящей работы заключается в установлении влияния циклических термических нагрузок на среднеквадратичные смещения атомов, как показателя прочности межатомных связей и микроповрежденности металла пароперегревательных труб.

Среднеквадратичные смещения атомов в элементарной кристаллической решетке и интегральные интенсивности дифракционных линий поликристаллического материала могут быть измерены методом рентгеновской дифракции, поэтому для реализации поставленной цели в качестве базового применен метод рентгенографии [38].

\section{Методика исследования и образцы}

Колебания атомов в кристаллической решетке реального кристалла весьма сложны, однако на основании рентгенометрии можно оценивать среднеквадратичные смещения атомов $\bar{U}^{2}$ при термическом или механическом циклическом деформировании [38, 39].

Методика настоящих исследований включала термоциклические испытания образцов № 1 и № 2, которые проводились с повышением температуры в каждом очередном температурном цикле.

Среднеквадратичные смещения атомов $\bar{U}^{2}$ рассчитывались по экспериментальным значениям интегральных интенсивностей дифракционных линий (110) образца и эталона на основании выражения (1) [38, 39]:

$$
\bar{U}^{2}=\frac{3}{4 \pi^{2}}\left(\frac{d}{n}\right)^{2} \cdot \ln \left(\frac{I}{I^{\prime}}\right), \AA^{2},
$$

где $n$ - порядок отражения; $d$ - межплоскостное расстояние, $\AA ; I^{\prime}$ - интегральная интенсивность для деформированного образца, имп.; $I$ - интегральная интенсивность для недеформированного образца (эталона), имп.

Величина среднеквадратичных смещений $\bar{U}^{2}$ связана с характеристической температурой Дебая $\Theta$ соотношением [40]:

$$
\bar{U}^{2}=\frac{3 h^{2} T}{k \cdot m \cdot \Theta^{2}} \rightarrow \Theta=\sqrt{\frac{3 h^{2} T}{k \cdot m \cdot \bar{U}^{2}}}, \mathrm{~K},
$$

где $h$ - постоянная Планка $\left(6,626070040(81) \cdot 10^{-34}\right.$ Дж·c); $k$ - постоянная Больцмана $\left(1,38064852(79) \cdot 10^{-23}\right.$ Дж/К); $m$ - масса атома (в данном случае $m_{\mathrm{Fe}}=9,2736 \cdot 10^{-23}$ г); $T$ - температура (295 K).

Из (2) видно, что $\bar{U}^{2} \sim 1 /\left(m \Theta^{2}\right)$. В то же время величина $\left(m \Theta^{2}\right)$ характеризует прочность межатомной связи, поскольку она пропорциональна силе $F$, возвращающей атомы в положение равновесия: $F \sim m \Theta^{2}$.

В экспериментах в качестве образца-свидетеля применялся специально подготовленный идеальномозаичный поликристаллический образец (эталон) из стали 12Х1МФ.

Интегральная интенсивность дифракционных линий определялась на рентгеновском дифрактометре методом набора импульсов как площадь под кривой профиля дифракционной линии.

Исследованию подвергались образцы № 1 и № 2, вырезанные из околошовных зон на расстоянии 8 мм слева и справа от оси однородного сварного узла, выполненного из низколегированной жаропрочной стали перлитного класса 12Х1МФ, согласно схеме на рис. 1 .

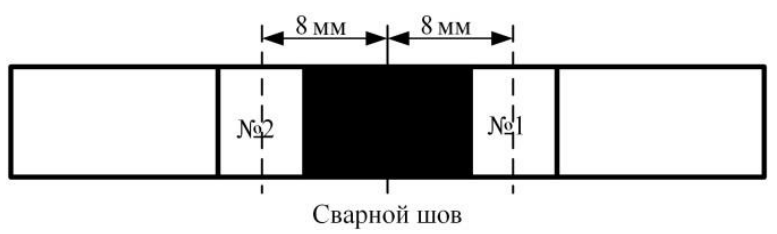

Рис. 1. Схема вырезки образиов

Fig. 1. Diagram of specimen cutout

Химический состав стали по массе [41]: 0,08-0,15 C; 0,9-1,2 Cr; 0,25-0,35 Mo; 0,15-0,30 V; $0,4-0,7 \mathrm{Mn} ; \quad 0,17-0,37 \mathrm{Si} ; \quad<0,25 \mathrm{Ni} ; \quad<0,20 \mathrm{Cu}$; $<0,025 \mathrm{~S} ;<0,030 \mathrm{P} ;$ остальное - железо.

Характер распределения интенсивности от угла дифракции профиля дифракционных линий и среднеквадратичных отклонений устанавливался методом рентгенографии с применением рентгеновского дифрактометра общего назначения типа ДРОН (Россия). Длина волны используемого излучения рентгеновской трубки составляла $\lambda_{\mathrm{CuK}_{\alpha_{\mathrm{cp}}}}=1,54178 \AA$.

Термоциклирование осуществлялось в электропечи МИМП-10УЭ (Россия).

Морфология поверхности контролировалась с помощью портативного электронного микроскопа типа PENSCKOPE (Taiwan) с увеличением $\times 10$. 


\section{Экспериментальные результаты}

Влияние процесса термоциклирования на амплитуду тепловых колебаний атомов $\bar{U}^{2}$

На рис. 2, 3 представлены экспериментальные результаты изменения субмикроскопических свойств III рода в процессе термоциклирования.
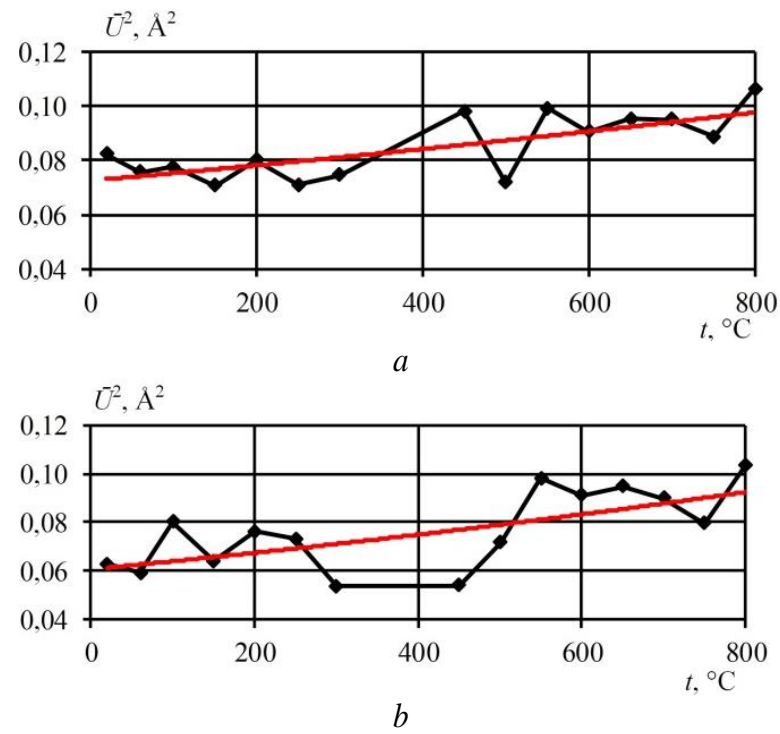

Pис. 2. Зависимость среднеквадратичных отклонений атомов от температуры термоциклирования в образие № 1: a) наружная сторона; b) внутренняя сторона

Fig. 2. Dependence of root-mean-square deviations $(R M S D)$ on thermal cycling temperature in specimen no. 1: a) external side; b) internal side

Значения среднеквадратичных отклонений атомов $\bar{U}^{2}$ изменяются от 0,08 до $0,105 \AA^{2}$ (образец № 1 , наружная сторона, рис. $2, a$ ) и от 0,06 до $0,105 \AA^{2}$ (образец № 1, внутренняя сторона, рис. $2, b$ ).
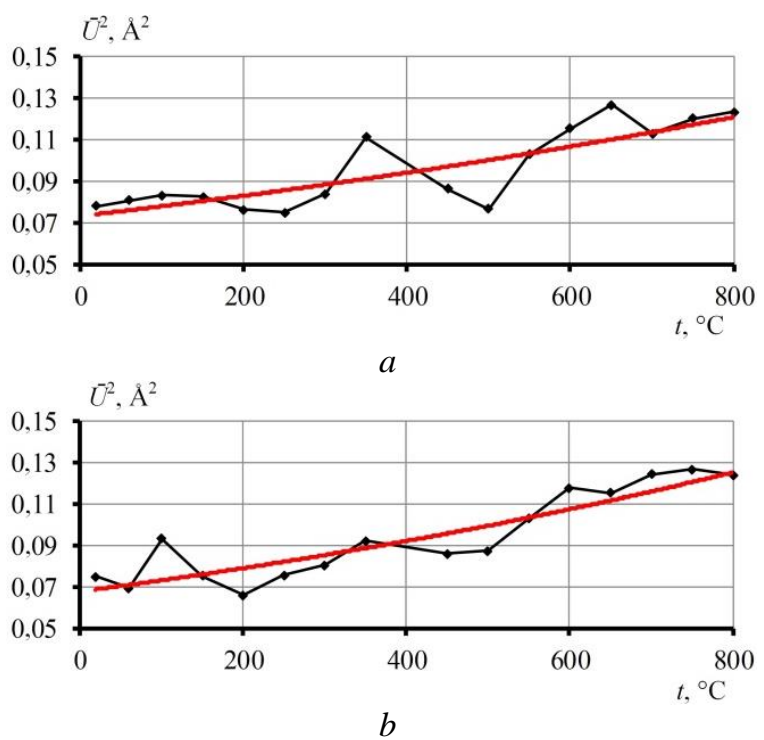

Pис. 3. Зависимость среднеквадратичных отклонений атомов от температуры термоциклирования в образие № 2: a) наружная сторона; b) внутренняя сторона

Fig. 3. Dependence of RMSD on thermal cycling temperature in specimen no. 2: a) external side; b) internal side
Для образца № 2 амплитуда среднеквадратичных смещений атомов $\bar{U}^{2}$ несколько больше и при максимальной температуре термоцикла $800{ }^{\circ} \mathrm{C}$ достигает значений $0,125 \AA^{2}$ (рис. 3).

Для всех кривых (рис. 2, 3) характерны значительные осцилляции $\bar{U}^{2}$ в процессе термоциклирования и отмечается общая тенденция к росту амплитуды колебаний при повышении температуры. Этот факт может быть положен в основу диагностики.

Наблюдаемые осцилляции могут быть объяснены наличием дефектов и неоднородностей в реальных поликристаллических материалах, в результате чего удельная внутренняя энергия неоднородно распределяется между атомными связями. Все существующие атомные связи не находятся в одинаковых условиях, при внешних возмущениях параллельно разрывам связей протекает процесс перераспределения дефектов и возникновение новых.

Если допустить, что атомы колеблются как связанные между собой маятники, то рост средней амплитуды колебаний можно отождествить с расшатыванием, ослаблением и разрывом связей, с увеличением в процессе термоциклирования общего количества изолированных маятников, то есть с началом и развитием процесса разрушения на субмикроуровне.

На основании изложенной модели допустимо предположить, что субмикроскопической характеристикой прочности могут служить среднеквадратичные отклонения атомов. Важно отметить, что наблюдаемые зависимости являются проявлением взаимодействия не всех атомов, а тех, которые находятся на поверхности в пределах «видимости» рентгеновского луча.

Если под влиянием внешней среды, например, при термоциклировании, изменяются условия взаимодействия атомов твердого тела, то изменяются и все характеристики, с этим связанные, что объясняет их осциллирующий характер.

Изменение условий взаимодействия атомов обусловливается не только температурой, но и неоднородностью дефектов структуры.

В процессе термоциклирования с ростом температуры растворимость газов атмосферного воздуха, основной естественной составляющей которого является азот, увеличивается. При этом адсорбция - это первый и необходимый шаг для проникновения газов внутрь.

Наличие сил, удерживающих молекулы на поверхности, обычно связывают с полярностью молекул, при которой центры разноименных электрических зарядов ядра и оболочки смещены друг относительно друга, создавая так называемый диполь. Если центры разноименных зарядов молекул не смещены относительно друг друга, молекулы неполярны. К неполярным молекулам относятся $\mathrm{O}_{2}, \mathrm{~N}_{2}, \mathrm{H}_{2}$, а также инертные газы - гелий, неон, ксенон, криптон. Если молекула газа в виде диполя приближается к твердой поверхности, имеющей ионную кристаллическую решетку, то под действием электрического поля, создаваемого поверхностными ионами адсорбента, полярные молекулы газа ориентируются соответствующим образом и остаются связанными поверхностным полем твердого тела. 
Если же к ионной кристаллической решетке на атомное расстояние приближается неполярная молекула, то картина процесса в целом не меняется. Речь в данном случае может идти только о прочности адсорбции.

И хоть явления адсорбции газов чрезвычайно сложны, изложенное все же позволяет представить взаимодействие между металлической решеткой и приблизившейся к ней полярной или неполярной молекулой в связи с общностью процессов адсорбции. Неполярная молекула может стать мгновенно полярной из-за колебаний и мгновенных смещений центров зарядов обоих знаков, происходящих в молекулах и приводящих к образованию временных диполей. При этом, совершая тепловые колебания с большой амплитудой, молекулы (атомы) газа могут проникать внутрь кристаллической решетки и занимать свободные узлы или места между узлами (рис. 4). Продолжая совершать тепловые колебания, они диффундируют на новые места, распространяясь по объему. Этот процесс известен как абсорбция. Процесс абсорбции тесно связан с диффузией и образованием твердых растворов внедрения в кристаллической решетке На рис. 4 представлены некоторые структуры, образованные атомами внедрения азота с кристаллической решеткой -железа.

Железо с азотом способно формировать фазы внедрения типа $\mathrm{Fe}_{4} \mathrm{~N}, \mathrm{Fe}_{2} \mathrm{~N}, \mathrm{Fe}_{3} \mathrm{~N}$. Нитриды также могут образовываться с хромом, марганцем, ванадием, молибденом, т. е. с элементами, обычно являющимися легирующими для сталей. Это $\mathrm{Cr}_{7} \mathrm{~N}, \mathrm{CrN}, \mathrm{Mn}_{4} \mathrm{~N}$, $\mathrm{Mn}_{2} \mathrm{~N}, \mathrm{Mn}_{3} \mathrm{~N}, \mathrm{VN}, \mathrm{V}_{2} \mathrm{~N}, \mathrm{MoN}, \mathrm{Mo}_{2} \mathrm{~N}$ и др.

Предварительно атомы азота распределяются статистически.

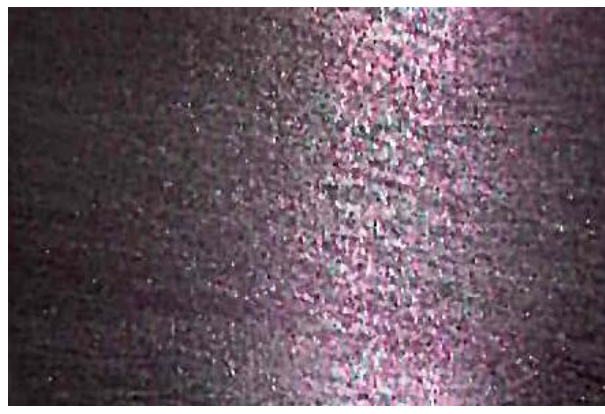

$a$

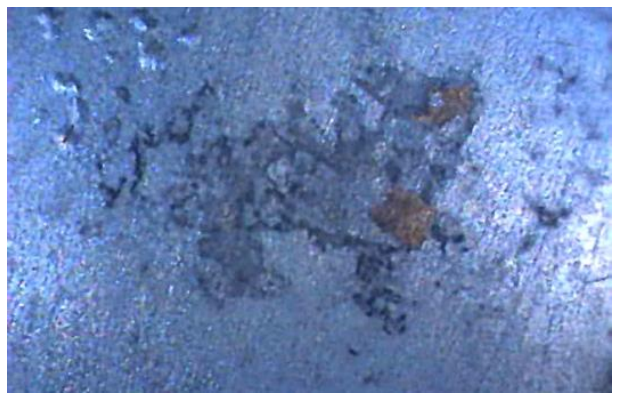

$c$
При образовании фаз внедрения атомы неметалла приобретают упорядоченность, располагаясь в октаили тетраэдрических пустотах растворителя (рис. 4).

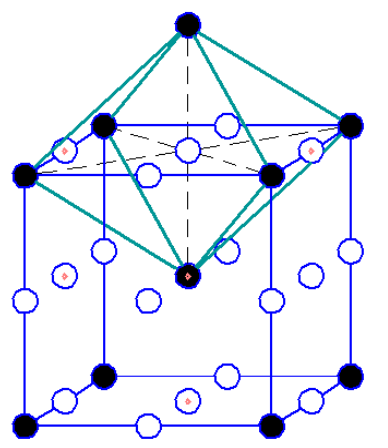

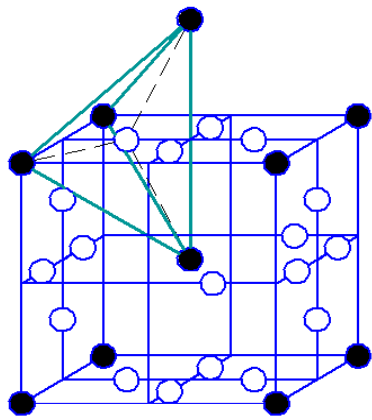

$b$
Pис. 4. Упорядоченное распределение азота в ОЦКрешетке железа: а) в октаэдрических позициях; b) в тетраэдрических позициях; - - атомы железа; о - атомы азота

Fig. 4. Ordered distribution of nitrogen in BCC-lattice of iron: a) in octahedral site; b) in tetrahedral site; $\bullet-$ iron atoms; 0 - nitrogen atoms

Первоначально происходит процесс насыщения поверхности и диффузионное проникновение атомов в объем. В зависимости от температуры и структуры образовавшихся фаз внедрения изменяются и цвета побежалости поверхности образца от коралловокрасного при температуре отжига $200{ }^{\circ} \mathrm{C}$ (рис. 5, a) до голубого при $250{ }^{\circ} \mathrm{C}$ и черно-зеленых и фиолетовых оттенков при более высоких температурах (рис. 5, $b-d$ ).

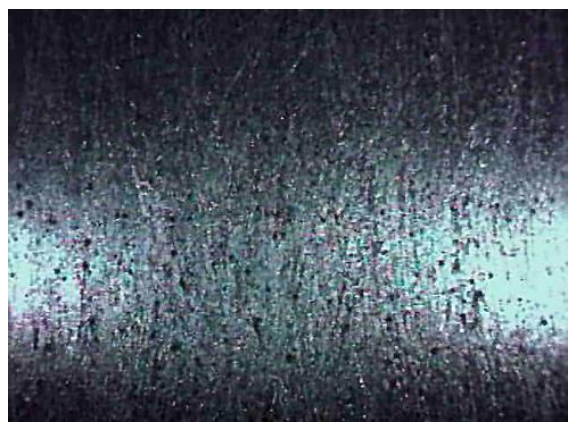

$b$

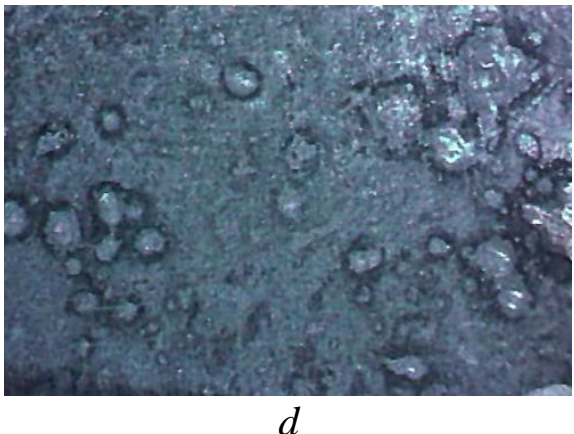

Pис. 5. Поверхность образиа при температурах отжига (х10): a) $200{ }^{\circ} \mathrm{C}$ (коралловый ивет); b) $300{ }^{\circ} \mathrm{C}$ (чернозеленый ивет); с) $450{ }^{\circ} \mathrm{C}$ (синий цвет); d) $600{ }^{\circ} \mathrm{C}$ (серо-зеленый цвет)

Fig. 5. Specimen surface at the annealing temperatures (x10): a) $200{ }^{\circ} \mathrm{C}$ (coral color); b) $300{ }^{\circ} \mathrm{C}$ (black-green color); c) $450{ }^{\circ} \mathrm{C}$ (blue color); d) $600{ }^{\circ} \mathrm{C}$ (grey-green color) 
Процесс проникновения сводится к замене вакансий атомами азота и диффузии вакансий к поверхности. В результате проникновения реализуется диффузионное насыщение азотом и одновременное «залечивание» вакансий в объеме путем их перемещения к поверхности, что проявляется в виде следов пористости и питтинга (рис. $5, b$ ).

При определенной концентрации атомов азота их статистическое распределение сменяется упорядоченным размещением с образованием пленок или мелкокристаллических кластеров нитридов по типу $\mathrm{Fe}_{4} \mathrm{~N}, \mathrm{Fe}_{2} \mathrm{~N}$ или $\mathrm{Fe}_{3} \mathrm{~N}$.

На рис. 5, $c, d$ видны следы шелушения и чешуйчатого отслоения на поверхности образца, формирование пузырьков газа и эрозия. Наблюдаемая картина напоминает последствия известных эффектов блистеринга и флекинга. В настоящем случае полагается, что наблюдаемая картина может быть связана не только с окислением поверхности, но и с внутриструктурной газовой пористостью и вакансионным замещением статистически распределенными атомами внедрения (азота), инициированным влиянием температуры.

Результатом процесса азотирования является перестройка решетки металла, которая становится более жесткой, примесь внедрения - азот - как бы цементирует ее. Металл в целом упрочняется.

Энергетическое состояние зон металла в состоянии нитрида изменяется в сторону увеличения связей между атомами и, соответственно, уменьшения амплитуд среднеквадратичных смещений атомов. Это подтверждается сопоставлением результатов термоциклирования образцов № 1 и № 2 (рис. 2,3 ) и холодного циклического деформирования образца (рис. 6), вырезанного из околошовной зоны с той же стороны сварного шва, что и образец № 1, результаты которого были опубликованы ранее данной статьи в [35] и помещены здесь для визуализации.

Сравнение показывает, что при холодном циклическом деформировании (рис. 6) среднеквадратичные отклонения изменяются в целом в более широких пределах. При внешних давлениях до 250 МПа амплитуда колебаний атомов составляет $0,125 \AA^{2}$. В диапазоне давлений 250-420 МПа среднеквадратичные отклонения уменьшаются до $0,09 \AA^{2}$, что можно связать с упрочнением материала за счет наклепа. Свыше давлений $420 \mathrm{MПа}$

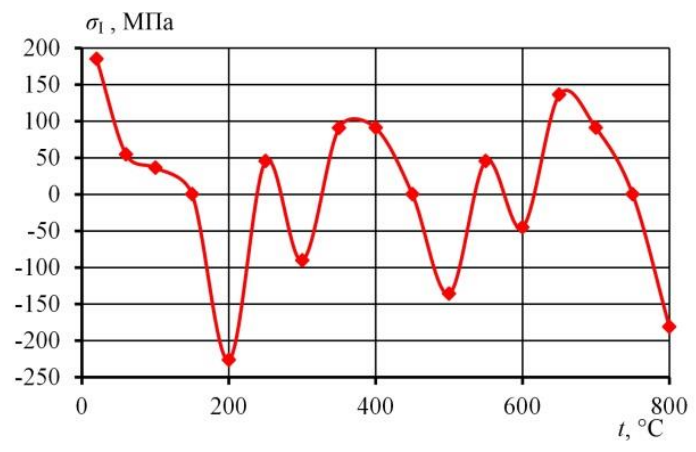

$a$ среднеквадратичные отклонения возрастают и достигают $0,2 \AA^{2}$ при давлениях $550 \mathrm{MПа}$, соответствующих $\sigma_{\text {в }}$ для данной стали $\left(\sigma_{\mathrm{B}}-\right.$ временное сопротивление разрушению). По справочным данным [41], при $20{ }^{\circ} \mathrm{C}$ $\sigma_{\mathrm{B}}=520 \mathrm{MПа}$, а $\sigma_{0,2}=330 \mathrm{MПа}$. Таким образом, видна вполне удовлетворительная корреляция между субмикрои макро-характеристиками прочности. С другой стороны, сопоставление рис. 2,3 с рис. 6 свидетельствует, что холодное циклическое деформирование быстрее приводит к разрыву межатомных связей и накоплению повреждаемости, а в результате обработки термоциклированием, наоборот, прочность межатомных связей возрастает, в результате чего материал упрочняется.

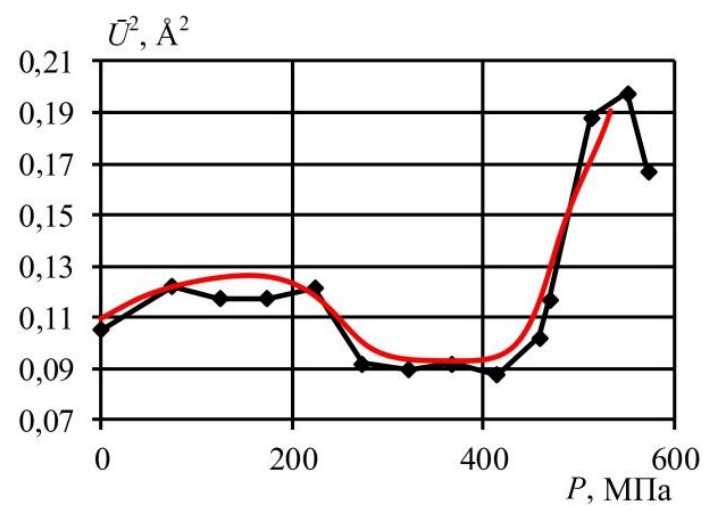

Pис. 6. Зависимость среднеквадратичных отклонений атомов от внешнего давления при холодном циклическом деформировании [35] (образеи из околотовной зоны со стороны образияа № 1)

Fig. 6. Dependence of RMSD on external pressure during cold cyclic strain [35] (a specimen from the weldaffected zone on the side of specimen no. 1)

Перераспределение зональных напряжений при термоциклировании

Зональные напряжения $\sigma_{\mathrm{I}}$, в отличие от субмикроскопических состояний, изображенных на рис. 2, 3, можно отнести к свойствам микроскопического уровня, обеспечиваемых прочностью зон, зерен, границ зерен или фаз.

Экспериментальные результаты перераспределения зональных напряжений при термоциклировании представлены на рис. 7.

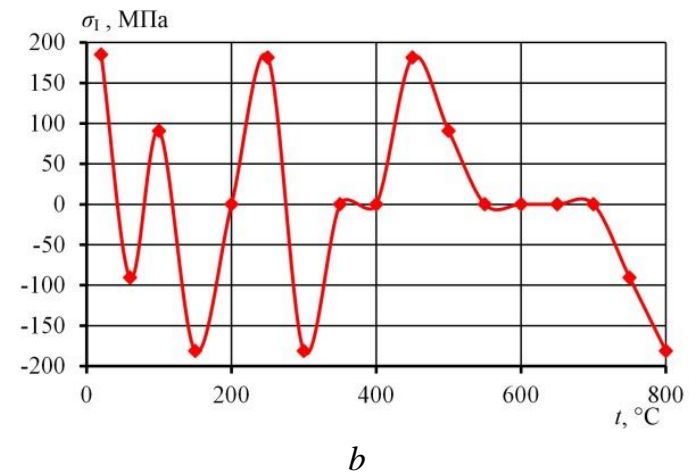

Рис. 7. Распределение зональных напряжений $\sigma_{I}$ при термоциклировании образиа № 1: a) внешняя сторона; b) внутренняя сторона

Fig. 7. Redistribution of zonal stresses at $\sigma_{I}$ thermal cycling of specimen no. 1: a) external side; $b$ ) internal side 
Основные особенности изменения внутренних напряжений I рода в процессе термоциклирования сводятся к следующему:

1. В зависимости от температуры наблюдаются осцилляции внутренних напряжений.

2. Внутренние напряжения перераспределяются от растягивающих напряжений $(+)$ до сжимающих (-) через релаксацию (обнуление) напряжений.

3. Релаксация напряжений является признаком ослабления и разрыва межатомных связей и сопровождает процесс разрушение на любой его стадии.

4. При внешних возмущающих нагрузках и при наличии градиентов температур длительные стабильные состояния в структуре материала отсутствуют в результате циклического перераспределения внутренних напряжений и свойств.

5. Внутренние напряжения по-разному изменяются на наружной и внутренней поверхностях, обнаруживая тем самым неравномерность распределения полей действующих напряжений по толщине стенки трубы. Полученные зависимости позволяют предварительно назначать наиболее благоприятную температуру эксплуатации. По данным рис. 7 самой оптимальной является температура $520{ }^{\circ} \mathrm{C}$, при которой на внутренней и внешней поверхностях устанавливаются скомпенсированные, незначительные по величине напряжения, равные порядка +50 МПа. Данная температура подтверждена опытом эксплуатации стали 12Х1МФ.

Осцилляция напряжений при термоциклировании может быть связана, кроме прочих причин (разнозернистость, разные значения коэффициента линейных расширений зерен и фаз, неоднородность химического состава и т. д.), и с предполагаемыми процессами азотирования. В азотированном слое могут присутствовать различные фазы внедрения по типу $\mathrm{Fe}-\mathrm{N}$ в соответствии с диаграммой состояния, температурой и концентрацией.

Кристаллическая структура фаз внедрения для легированных сталей достаточно многообразна и определяется соотношением $r=r_{x} / r_{m}$, где $r_{x}$ и $r_{m}-$ ионные радиусы внедренных атомов и атомов растворителя. При $r<0,59$ образуются гранецентрированная кубическая, гексагональная плотноупакованная, объемноцентрированная кубическая и простая гексагональная структуры. При $r>0,59$ формируется более сложная структура фаз внедрения. Это исходит из соображений устойчивости связей «металл-неметалл» - размер междоузлия должен быть меньше размера внедряемого

\section{СПИСОК ЛИТЕРАТУРЫ}

1. Антикайн П.А. Обеспечение надежной эксплуатации трубопроводов тепловых электростанций // Теплоэнергетика. 2000. - № 4. - C. 2-5.

2. Failure analysis of boiler economizer tubes at power house / U. Pal, K. Kishore, S. Mukhopadhyay, G. Mukhopadhyay, S. Bhattacharya // Engineering Failure Analysis. - 2019. - V. 104. - P. 1203-1210.

3. The strength and the service life of power-generating equipment / A.V. Sudakov, A.I. Levchenko, I.A. Danyushevskii, B.N. Ivanov, L.L. Smelko, E.Y. Nefed'ev // Thermal Engineering. - 2003. V. 150. - № 2. - P. 133-140.

4. Failure analysis of a steam pipe weld used in power generation plant / Q. Chu, M. Zhang, J. Li, Y. Chen, H. Luo, Q. Wang // Engineering Failure Analysis. - 2014. - V. 44. - P. 363-370. атома, что обеспечит более устойчивые межатомные связи. Если размер междоузлия и дальше будет уменьшаться, то может произойти потеря устойчивости связей «металл-металл» за счет деформации кристаллической решетки металла. Чтобы не допустить этого, происходит формирование более сложных структур фаз внедрения, что можно расценить как реакцию системы на возможное увеличение деформации кристаллической решетки металла.

Таким образом, физический механизм наблюдаемых осцилляций внутренних напряжений сводится к организации сложных связей «металл-неметалл», «металл-металл» и т. д., направленных на поддержание устойчивости системы.

Процесс осцилляций напряжений поддерживается, кроме того, тем, что и распределение азота по глубине слоя при азотировании также имеет скачкообразный характер вследствие неравномерности насыщения слоев азотом.

\section{Выводы}

1. Экспериментально установлено влияние циклических термических нагрузок на среднеквадратичные смещения атомов.

2. Показано, что в результате термического влияния прочность межатомных связей увеличивается.

3. Проиллюстрирована корреляция между субмикрои макроскопическими характеристиками прочности и показано, что амплитуда тепловых колебаний атомов, являясь характеристикой прочности межатомных связей, может быть диагностическим признаком накопления и развития повреждаемости металла.

4. Уменышение амплитуды среднеквадратичных смещений при термоциклировании объяснено на основе возникновения твердых растворов замещения в результате процесса азотирования поверхности молекулярным азотом атмосферного воздуха

5. Одним из механизмов осцилляции внутренних напряжений I рода может быть процесс азотирования поверхности при термоциклировании, приводящий в многокомпонентных легированных сталях к организации сложных связей «металлнеметалл», направленных на поддержание устойчивости системы твердых растворов.

Работа выполнена при поддержке РФФИ «Фундаментальные основы инженерных наук (проект № 18-08-01265).

5. Failure analysis of branch connection on the reactor primary pipeline / L. Zhao, F. Chu, K. Luo, G. Li, W. Wang, S. Tu // Engineering Failure Analysis. - 2018. - V. 85. - P. 137-148.

6. Jalali A., Amiri Delouei A. Failure analysis in a steam turbine stop valve of a thermal power plant // Engineering Failure Analysis. 2019. - V. 105. - P. 1131-1140.

7. Savchenko V.A. Several conceptual questions on lifetime management of Russian power-generating units at nuclear power stations // Thermal Engineering. - 2000. - V. 47. - № 5. - P. 375-382.

8. Popov A.B., Perevalova E.K., Sverchkov A.Y. The problem of prolonging the service life of thermal power equipment at power stations // Thermal Engineering. - 2003. - V. 50. - № 4. - P. 294-301.

9. Злепко В.Ф., Гринь Е.А., Швецова Т.А. Техническое перевооружение действующих ТЭС с учётом состояния металла // Электрические станции. - 2001. - № 7. - С. 12-18. 
10. Collini L., Giglio M., Garziera R. Thermomechanical stress analysis of dissimilar welded joints in pipe supports: Structural assessment and design optimization // Engineering Failure Analysis. - 2012. - V. 26. - P. 31-49.

11. Tumanovskii A.G., Rezinskikh V.F. The strategy of prolonging the service life and the technical reequipment of thermal power stations // Thermal Engineering. - 2001. - V. 48. - № 6. - P. 431-439.

12. Dolzhanskii P.R. Peculiarities of assessing the remaining service life of steam pipelines for operation beyond the fleet resource / Thermal Engineering. - 2005. - V. 52. - № 8. - P. 625-629.

13. Selection and analysis of material for boiler pipes in a steam plant / V. Lazić，D. Arsić， R.R. Nikolić，D. Rakić， S. Aleksandrović, M. Djordjević, B. Hadzima // Procedia Engineering. - 2016. V. 149. - P. 216-223.

14. Dubov A.A. Problems in estimating the remaining life of aging equipment // Thermal Engineering. - 2003. - V. 50. - № 11. P. 935-938.

15. Реконструкция паровых турбин ПТ-60-12,8 с восстановлением ресурса и повышением технико-экономических показателей В.В. Ермолаев, Н.Н. Гудков, А.Ю. Сосновский, С.А. Кошелев, А.Н. Бабиев, М.В. Бакурадзе, Д.Е. Губанов, А.И. Шкляр // Теплоэнергетика. - 2007. - № 4. - С. 28-31.

16. Effects of prior damage on the creep failure behaviour of similar and dissimilar welded $\mathrm{CrMoV}$ main steam pipes incorporating a partial repair / W. Sun, T.H. Hyde, J.A. Williams, A.A. Becker // International Journal of Pressure Vessels and Piping. - 2009. V. 86. - № 10. - P. 699-710.

17. Жакупов Г.А., Киселёв Л.А. Восстановление рабочих лопаток паровых турбин в условиях электростанций // Энергетик. 2007. - № 12. - C. $28-30$

18. Estimation of remaining service life taking microdamage into account / I.A. Danyushevskii, E.B. Kuprii, M.R. Malkin, E.A. Grin' // Thermal Engineering. - 2008. - V. 55. - P. 112-115.

19. Low cycle fatigue and creep fatigue interaction behavior of $9 \mathrm{Cr}-$ $0.5 \mathrm{Mo}-1.8 \mathrm{~W}-\mathrm{V}-\mathrm{Nb}$ heat-resistant steel at high temperature / X. Wang, W. Zhang, J. Gong, M.A. Wahab // Journal of Nuclear Materials. - 2018. - V. 505. - P. 73-84.

20. Structural criteria of recovery annealing regime selection for vver1000 reactor pressure vessel materials / B.A. Gurovich, Y.I. Shtrombakch, E.A. Kuleshova, S.V. Fedotova // Problems of Atomic Science and Technology. - 2010. - № 5. - P. 50-57.

21. A novel creep-fatigue interaction damage model with the stress effect to simulate the creep-fatigue crack growth behavior / $\mathrm{L}$. Xu, L. Zhao, Z. Gao, Y. Han // International Journal of Mechanical Sciences. - 2017. - V. 130. - P. 143-153.

22. Исследование закономерностей деформирования теплоустойчивых сталей в условиях ползучести при сложном напряженном состоянии / Ф.Ф. Гигиняк, П.А. Булах, В.Н. Можаровский, Т.Н. Можаровская // Проблемы прочности. - 2010. - № 4. C. $62-70$.

23. Microstructural evolution during creep of $316 \mathrm{LN}$ stainless steel multi-pass weld joints / V.D. Vijayanand, K. Laha, P. Parameswaran, V. Ganesan, M.D. Mathew // Materials Science and Engineering: A. - 2014. - V. 607. - P. 138-144.

24. Хапонен Н.А., Шевченко П.Н., Р Рассохин Г.И. Микроповрежденность как критерий оценки состояния металла и остаточного ресурса паропроводов ТЭС // Безопасность труда в промышленности. - 2004. - № 5. - С. 42-44.

25. Мороз Л.С. Механика и физика деформаций и разрушения материалов. - Л.: Машиностроение, 1984. - 224 с.

\section{Информация об авторах}

Заворин $\boldsymbol{A}$.C., доктор технических наук, профессор научно-образовательного центра И.Н. Бутакова Инженерной школы энергетики Национального исследовательского Томского политехнического университета.

Любимова Л.Л., кандидат технических наук, доцент научно-образовательного центра И.Н. Бутакова Инженерной школы энергетики Национального исследовательского Томского политехнического университета.

Буваков К.В., кандидат технических наук, доцент научно-образовательного центра И.Н. Бутакова Инженерной школы энергетики Национального исследовательского Томского политехнического университета.

Ташльков А.А., кандидат технических наук, доцент научно-образовательного центра И.Н. Бутакова Инженерной школы энергетики Национального исследовательского Томского политехнического университета.

Фисенко P.Н., инженер организационного отдела Инженерной школы энергетики Национального исследовательского Томского политехнического университета.
26. Иванов А.Н., Ягодкин ЮД.Применение дифракционных ловедение и термическая обработка металгов - 2000 - № 8 C. $11-15$. струкуры металлов рентгеновскими методами // МеталловеC. $7-10$.

. Rybakova L.M. Mechanical properties and substructure of metals // Science and Heat Treatment. - 1994. - V. 36. - P. 507-513. embrittlement of chromium-nickel steel and nickel alloys / S.N. Votinov, V.P. Kondrat'ev, V.N. Rechitskij, T.A. Krasina, V.P. Kolotushkin // Физика и химия обработки материалов. - № 1. - C. 19-26.

. Thomson R.M. Physics of solids. - New York: A.C. Batista // Journal of Alloys and Compounds. - 2015. V. 649. - P. 1246-1252. ность в твердом теле. - Новосибирск: Наука, 1986. - 176 с.

3. Влияние остаточных напряжений в зонах сварного узла на Л.Л. Любимова, К.В. Буваков, А.С. Кулеш, А.А. Ташлыков, Р.Н. Кулеш // Известия Томского политехнического университета. Инжиниринг георесурсов. - 2018. - Т. 329. - № 10. $128-142$ Y. Jin, Y. Zhao, X Yang H. Zhao, P. Han // Journal of Alloys and Compounds. - 2015. - V. 621. - P. 383-388.

роперегревателя / А.С. Заворин, Л Л. Любимова, А.А. Ташлыков, К.В. Буваков // Известия Томского политехнического университета. Инжиниринг георесурсов. - 2018. - Т. 329. - № 11. $124-135$

metal of convection superheat operation // Metal Science and Heat Treatment. - 2011. - V. 53. P. 136-140

Use of a magnetization effect for monitoring thermal inomogeneities in steam superheaters made of $12 \mathrm{Kh} 18 \mathrm{~N} 12 \mathrm{~T}$ steel $/$ V. Bogachev, V.I Sanakina, S.N. Starchikov, II Timonin // Power Technology and Engineering. - 2008. - V. 42. - № 1. 50-54.

. Русаков А.А. Рентгенография металлов. - М.: Атомиздат, - $480 \mathrm{c}$

Горелик С.С., Скаков Ю.А., Расторгуев Л.Н. Рентгенографи-

science - Cambridge: Cambridge University Press, 1994 - $586 \mathrm{p}$.

1. Масленков С.Б., Масленкова Е.А. Стали и сплавы для высоких температур: Справочник в двух книгах. - М.: Металлургия, 1991. - 382 с.

Поступила 22.02.2020 2. 
UDC 621.78:669.14-022.532

\title{
ASPECTS OF EVOLUTION OF METAL NANOSCALE CHARACTERISTICS DURING THERMAL CYCLING
}

\author{
Alexander S. Zavorin 1 , \\ zavorin@tpu.ru \\ Lyudmila L. Lyubimova ${ }^{1}$, \\ III@tpu.ru \\ Konstantin V. Buvakov1, \\ buvakov@tpu.ru \\ Alexander A. Tashlykov', \\ tashlykov@tpu.ru \\ Roman N. Fisenko', \\ ronifis@tpu.ru \\ ${ }^{1}$ National Research Tomsk Polytechnic University, \\ 30, Lenin avenue, Tomsk, 634050, Russia.
}

Relevance of the study is caused by the absence of experimental data, establishing a relation between submicroscopic (the third-order properties) and macroscopic properties of the first-order. Such interrelatedness, being a factor affecting the microstructure-strength ratio, can considerably facilitate a further success in solving the issues of life extension.

The aim of the study is to establish the effect of cyclic thermal loads on the root-mean-square displacements of atoms as a sign of interatomic bond strength and microdamage of metal of superheater tubes.

Object of study are specimens of heat-resistant pearlitic 12Cr1MoV steel from the weld-affected zone.

Methods include physical simulation of operation conditions by thermal cycling of specimens in the MIMP-10UE furnace, X-ray dosimetry of specimens, evaluation of internal structural stresses using X-ray diffractometer DRON, and surface morphology by means of PENSCKOPE microscope.

Results of the study established that thermal cyclic loads have effect on the root-mean-square displacements of atoms. It was shown that thermal effect results in increase of the strength of interatomic bonds. The paper demonstrates the correlation between submicro- and macroscopic characteristics of strength, as well as the fact that the amplitude of atomic thermal vibrations, being a characteristic of interatomic bonds strength, can be a diagnostic indicator of accumulation and development of metal damaging. Decrease of root-meansquare displacements amplitude during thermal cycling is explained by the hypothesis about occurrence of substitution solid solutions as a result of surface nitriding by molecular nitrogen of the atmospheric air. Under this hypothesis it was proved that one of oscillation mechanisms for internal stresses of the first-kind can be the process of surface nitriding during thermal cycling, which results in complex bonds between metal and nonmetal in the multicomponent alloyed steels, aimed at maintaining the stability of solid solution system.

\section{Key words:}

Weld seam, weld-affected zone, root-mean-square deviation of atoms, internal stress, thermal cycling.

The research was supported by the RFBR «Fundamental bases of engineering sciences» (project no. 18-08-01265).

\section{REFERENCES}

1. Antikayn P.A. Obespecheniye nadezhnoy ekspluatatsii truboprovodov teplovykh elektrostantsiy [Provision of reliable pipeline operation at thermal power stations]. Teploenergetika, 2000, no. 4, pp. $2-5$.

2. Pal U., Kishore K., Mukhopadhyay S., Mukhopadhyay G., Bhattacharya S. Failure analysis of boiler economizer tubes at power house. Engineering Failure Analysis, 2019, vol. 104, pp. 1203-1210.

3. Sudakov A.V., Levchenko A.I., Danyushevskii I.A., Ivanov B.N., Smelko L.L., Nefed'ev E.Y. The strength and the service life of power-generating equipment. Thermal Engineering, 2003, vol. 150 , no. 2, pp. 133-140.

4. Chu Q., Zhang M., Li J., Chen Y., Luo H., Wang Q. Failure analysis of a steam pipe weld used in power generation plant. Engineering Failure Analysis, 2014, vol. 44, pp. 363-370.

5. Zhao L., Chu F., Luo K., Li G., Wang W., Tu S. Failure analysis of branch connection on the reactor primary pipeline. Engineering Failure Analysis, 2018, vol. 85, pp. 137-148.
6. Jalali A., Amiri Delouei A. Failure analysis in a steam turbine stop valve of a thermal power plant. Engineering Failure Analysis, 2019, vol. 105, pp. 1131-1140.

7. Savchenko V.A. Several conceptual questions on lifetime management of Russian power-generating units at nuclear power stations. Thermal Engineering, 2000, vol. 47, no. 5, pp. 375-382.

8. Popov A.B., Perevalova E.K., Sverchkov A.Y. The problem of prolonging the service life of thermal power equipment at power stations. Thermal Engineering, 2003, vol. 50, no. 4, pp. 294-301.

9. Zlepko V.F., Grin' E.A., Shvetsova T.A. Tekhnicheskoye perevooruzheniye deystvuyushchikh TES s uchotom sostoyaniya metalla [Technical reequipment of effective TPS with consideration for metal condition]. Elektricheskiye Stantsii, 2001, no. 7 , pp. $12-18$.

10. Collini L., Giglio M., Garziera R. Thermomechanical stress analysis of dissimilar welded joints in pipe supports: Structural assessment and design optimization. Engineering Failure Analysis, 2012, vol. 26, pp. 31-49.

11. Tumanovskii A.G., Rezinskikh V.F. The strategy of prolonging the service life and the technical reequipment of thermal power stations. Thermal Engineering, 2001, vol. 48, no. 6, pp. 431-439. 
12. Dolzhanskii P.R. Peculiarities of assessing the remaining service life of steam pipelines for operation beyond the fleet resource. Thermal Engineering, 2005, vol. 52, no. 8, pp. 625-629.

13. Lazić V., Arsić D., Nikolić R.R., Rakić D., Aleksandrović S., Djordjević M., Hadzima B. Selection and analysis of material for boiler pipes in a steam plant. Procedia Engineering, 2016, vol. 149, pp. 216-223.

14. Dubov A.A. Problems in estimating the remaining life of aging equipment. Thermal Engineering, 2003, vol. 50, no. 11, pp. 935-938.

15. Ermolaev V.V., Gudkov N.N., Sosnovskiy A.Y., Koshelev S.A., Babiev A.N., Bakuradze M.V., Gubanov D.E., Shklyar A.I. Rekonstruktsiya parovykh turbin PT-60-12,8 s vosstanovleniem resursa i povysheniem tekhniko-ekonomicheskikh pokazateley [Reconstruction of steam turbines PT-60-12.8 with operational life extending and performance increase]. Teploenergetika, 2007, no. 4, pp. 28-31.

16. Sun W., Hyde T.H., Williams J.A., Becker A.A. Effects of prior damage on the creep failure behaviour of similar and dissimilar welded $\mathrm{CrMoV}$ main steam pipes incorporating a partial repair. International Journal of Pressure Vessels and Piping, 2009, vol. 86, no. 10, pp. 699-710.

17. Zhakupov G.A., Kiselev L.A. Vosstanovlenie rabochikh lopatok parovykh turbin $\mathrm{v}$ usloviyakh elektrostantsiy [Reconditioning of steam turbine rotor blades under the conditions of electric power plants]. Energetik, 2007, no. 12, pp. 28-30.

18. Danyushevskii I.A., Kuprii E.B., Malkin M.R., Grin' E.A. Estimation of remaining service life taking microdamage into account. Thermal Engineering, 2008, vol. 55, pp. 112-115.

19. Wang X., Zhang W., Gong J., Wahab M.A. Low cycle fatigue and creep fatigue interaction behavior of $9 \mathrm{Cr}-0.5 \mathrm{Mo}-1.8 \mathrm{~W}-\mathrm{V}-\mathrm{Nb}$ heatresistant steel at high temperature. Journal of Nuclear Materials, 2018, vol. 505, pp. 73-84.

20. Gurovich B.A., Shtrombakch Y.I., Kuleshova E.A., Fedotova S.V. Structural criteria of recovery annealing regime selection for vver1000 reactor pressure vessel materials. Problems of Atomic Science and Technology, 2010, no. 5, pp. 50-57.

21. Xu L., Zhao L., Gao Z., Han Y. A novel creep-fatigue interaction damage model with the stress effect to simulate the creep-fatigue crack growth behavior. International Journal of Mechanical Sciences, 2017, vol. 130, pp. 143-153.

22. Giginyak F.F., Bulakh P.A., Mozharovskiy V.N., Mozharovskaya T.N. Issledovanie zakonomernostey deformirovaniya teploustoychivykh staley $\mathrm{v}$ usloviyakh polzuchesti pri slozhnom napryazhennom sostoyanii [Research of heat resistant steels deformation pattern under the conditions of creeping in a combined stress state]. Problemy Prochnosti, 2010, no. 4 , pp. $62-70$

23. Vijayanand V.D., Laha K., Parameswaran P., Ganesan V., Mathew M.D. Microstructural evolution during creep of 316LN stainless steel multi-pass weld joints. Materials Science and Engineering: A, 2014. vol. 607, pp. 138-144.

24. Khaponen N.A., Shevchenko P.N., Rassokhin G.I. Mikropovrezhdennost kak kriteriy otsenki sostoyaniya metalla i ostatochnogo resursa paroprovodov TES [Microdamage as a criterion for evaluaiton of metal condition and remaining life of TPS steam lines]. Bezopasnost' Truda v Promyshlennosti, 2004, no. 5, pp. $42-44$.

25. Moroz L.S. Mekhanika i fizika deformatsiy $i$ razrusheniya materialov [Mechanics and physics of deformation and fracture of materials]. Leningrad, Mashinostroenie Publ., 1984. 224 p.
26. Ivanov A.N., Yagodkin Y.D. Primenenie difraktsionnykh metodov dlya tekhnologicheskogo kontrolya materialov [Application of diffraction methods for in-process monitoring of materials]. Metallovedeniye i Termicheskaya Obrabotka Metallov, 2000, no. 8, pp. 11-15.

27. Ivanov A.N., Klimanek P., Polyakov A.M. Issledovanie substruktury metallov rentgenovskimi metodami [Study of metal substructure by X-ray methods]. Metallovedeniye i Termicheskaya Obrabotka Metallov, 2000, no. 8, pp. 7-10.

28. Rybakova L.M. Mechanical properties and substructure of metals. Metal Science and Heat Treatment, 1994, vol. 36, pp. 507-513.

29. Votinov S.N., Kondrat'ev V.P., Rechitskij V.N., Krasina T.A., Kolotushkin V.P. The role of the structure in radiation strengthening and embrittlement of chromium-nickel steel and nickel alloys. Fizika i khimiya obrabotki materialov, 2002, no. 1, pp. 19-26.

30. Wert C.A., Thomson R.M. Physics of solids. New York, McGrawHill, $1964.436 \mathrm{p}$.

31. Dubiel S.M., Costa B.F.O., Cieslak J., Batista A.C. Debye temperature of nanocrystalline $\mathrm{Fe}-\mathrm{Cr}$ alloys obtained by mechanical alloying. Journal of Alloys and Compounds, 2015, vol. 649, pp. 1246-1252.

32. Gabuda S.P., Lundin A.G., Fedin E.I. Vnutrennyaya podvizhnost v tverdom tele [Intrinsic mobility in solid bodies]. Novosibirsk, Nauka Publ., 1986. 176 p.

33. Zavorin A.S., Lyubimova L.L., Buvakov K.V., Kulesh A.S., Tashlykov A.A., Kulesh R.N. Influence of residual stresses on resistance to brittle fracture in weldment zones. Bulletin of the Tomsk Polytechnic University. Geo Assets Engineering, 2018, vol. 329, no. 10, pp. 128-142. In Rus.

34. Qi L., Jin Y., Zhao Y., Yang X., Zhao H., Han P. The structural, elastic, electronic properties and Debye temperature of Ni3Mo under pressure from first-principles. Journal of Alloys and Compounds, 2015, vol. 621, pp. 383-388.

35. Zavorin A.S., Lyubimova L.L., Tashlykov A.A., Buvakov K.V. Structural aspects of cyclical strength of a superheater weldment. Bulletin of the Tomsk Polytechnic University. Geo Assets Engineering, 2018, vol. 329, no. 11, pp. 124-135. In Rus.

36. Priymak E.Y., Gryzunov V.I. Special features of behavior of the metal of convection superheater in the process of high-temperature operation. Metal Science and Heat Treatment, 2011, vol. 53, pp. 136-140.

37. Bogachev V.A., Sanakina V.I., Starchikov S.N., Timonin I.L. Use of a magnetization effect for monitoring thermal inhomogeneities in steam superheaters made of $12 \mathrm{Kh} 18 \mathrm{~N} 12 \mathrm{~T}$ steel. Power Technology and Engineering, 2008, vol. 42, no. 1, pp. 50-54.

38. Rusakov A.A. Rentgenografiya metallov [X-ray analysis for metals]. Moscow, Atomizdat Publ., 1977. 480 p.

39. Gorelik S.S., Skakov Y.A., Rastorguev L.N. Rentgenograficheskiy $i$ elektronno-opticheskiy analiz [X-ray and electron-optical analysis]. Moscow, MISIS Publ., 2002. 358 p.

40. Woodruff D.P., Delchart T.A. Modern techniques of surface science. Cambridge, Cambridge University Press, 1994. 586 p.

41. Maslenkov S.B., Maslenkova E.A. Stali $i$ splavy dlya vysokikh temperatur [Steel and alloys for high temperatures]. Moscow, Metallurgiya Publ., 1991. 382 p.

Received: 22 February 2020.

Information about the authors

Alexander S. Zavorin, Dr. Sc., professor, National Research Tomsk Polytechnic University.

Lyudmila L. Lyubimova, Cand. Sc., associate professor, National Research Tomsk Polytechnic University.

Konstantin V. Buvakov, Cand. Sc., associate professor, National Research Tomsk Polytechnic University.

Alexander A. Tashlykov, Cand. Sc., associate professor, National Research Tomsk Polytechnic University.

Roman N. Fisenko, engineer, National Research Tomsk Polytechnic University. 